\title{
Hacia un enfoque contextual-existencial del suicidio: recomendaciones para la prevención
}

\section{Towards a contextual-existential approach to suicide: recommendations for prevention}

\author{
Marta González González ${ }^{1}$, Juan M. García-Haro², Henar García-Pascual², \\ Mónica T. Sánchez Pérez ${ }^{3}$, Sara Barrio-Martínez y Jairo Voces Oviedo ${ }^{4}$ \\ ${ }^{1}$ Red de Salud Mental de Araba. País Vasco, España \\ ${ }^{2}$ Servicio de Salud del Principado de Asturias. Asturias, España \\ ${ }^{3}$ Servicio Madrileño de Salud. Madrid, España \\ ${ }^{4}$ Instituto de Investigación Marqués de Valdecilla (IDIVAL). Cantabria, España
}

\begin{abstract}
Resumen: Existen diferentes modelos conceptuales de pensar el suicidio. Se presenta una visión contextual-existencial del suicidio y se proponen tres características distintivas: abierta, plural y dinámico-fluctuante. Para su presentación se utiliza la metáfora del drama existencial y la figura del iceberg. A la luz de este nuevo enfoque del suicidio se proporcionan algunas recomendaciones específicas para la dirección futura de la prevención. Se cierra el texto con un resumen de las principales conclusiones.

Palabras clave: suicidio, fenomenología, prevención, factores de protección, prevención del suicidio.

Abstract: There are different conceptual models of thinking about suicide. A contextual-existential vision of suicide is presented, and three distinctive characteristics are proposed: open, plural and dynamic-fluctuating. The metaphor of existential drama and the figure of the iceberg are used to present this vision. In the light of this new approach to suicide,
\end{abstract}

Para citar este artículo: González González, M., García-Haro, J. M., García-Pascual, H., Sánchez Pérez, M. T., Barrio-Martínez, S. y Voces Oviedo, Jairo (2021). Hacia un enfoque contextual-existencial del suicidio: recomendaciones para la prevención. Clínica Contemporánea, 12(1), Artículo e1. https://doi.org/10.5093/cc2021a3

\footnotetext{
Marta González González. Es Facultativo Especialista de Área (FEA) en Psicología Clínica en la Red de Salud Mental de Araba. Osakidetza. País Vasco.

Juan M. García-Haro. Es Facultativo Especialista de Área (FEA) en Psicología Clínica en el Centro de Salud Mental de Adultos de Pola de Siero. Asturias. Servicio de Salud del Principado de Asturias.

Henar García-Pascual. Es Enfermera Especialista en Salud Mental en el Servicio de Salud del Principado de Asturias.

Mónica T. Sánchez Pérez. Es Enfermera Especialista en Salud Mental en el Servicio Madrileño de Salud. Madrid.

Sara Barrio-Martínez. Es Psicóloga General Sanitaria. Investigadora en el Instituto de Investigación Marqués de Valdecilla (IDIVAL) de Cantabria.

Jairo Voces Oviedo. Enfermero y Graduado en psicología en el Servicio de Salud del Principado de Asturias.
}

La correspondencia sobre este artículo debe enviarse a la primera autora al e-mail: martagg118@gmail.com

(cc) BY-NC-ND Este es un artículo Open Access bajo la licencia CC BY-NC-ND. 
some specific recommendations for the future direction of prevention are provided. The text closes with a summary of the main conclusions.

Keywords: suicide, phenomenology, prevention, preventive factors, suicide prevention

Antes de preguntarse qué investigar y cómo (Cha et al., 2018), habría que preguntarse qué es el suicidio y cómo se manifiesta (ontología y fenomenológica).

En este sentido existen al menos dos modelos bien diferenciados en la literatura clínica para la comprensión del suicidio: el modelo biomédico o diagnosticocéntrico y el modelo contextual-fenomenológico o transdiagnóstico (García-Haro et al., 2018; González González et al., 2019; García-Haro, García-Pascual, González González, Barrio-Martínez y García-Pascual, 2020). Cada uno pone en marcha y posibilita un sistema de evaluación, ayuda y de prevención diferentes.

Desde una perspectiva biomédica, el suicidio y las conductas suicidas se entienden como "síntomas" de un trastorno mental (se refiere aquí a la depresión y a los trastornos límites de la personalidad), o como una consecuencia, complicación o evolución "natural" de una enfermedad mental (véase la "depresión resistente") o incluso como una enfermedad psiquiátrica en sí misma -véase la propuesta del Trastorno del comportamiento suicida incluida en la sección III "Condiciones para la futura investigación" del Diagnostic and Statistical Manual of Mental Disorders (DSM-5, por sus siglas en inglés) (APA, 2013). Para una revisión de los supuestos, prácticas, limitaciones y riesgos de este modelo en los Planes, Estrategias y/o Protocolos, según se aplican en el sistema sanitario español, véase García-Haro, García-Pascual, González González, Sánchez Pérez y Barrio-Martínez (2020).

Desde una perspectiva contextual-existencial, el suicidio se vería como una solución límite a una crisis vital de un sujeto con capacidad de acción-decisión-ejecución que, en una circunstancia determinada -construida como insufrible, irresoluble, interminable, inescapable, sin futuro y sin esperanza-, decide quitarse la vida, pudiendo elegir no hacerlo. Este "pudiendo elegir no hacerlo" es fundamental, pues sin él, el suicidio carece de sentido, quedaría reducido a reacción o síntoma, pero no a acción humana (acto conductual con sentido e intencionalidad).

El objetivo de este artículo es presentar el modelo contextual-existencial del suicidio. Se revisan tres de sus características más distintivas: abierta, plural y dinámico-fluctuante. Para ilustrar este modelo se utiliza la metáfora del drama existencial y la figura del iceberg. A la luz de este nuevo enfoque, se proporcionan algunas recomendaciones específicas para la dirección futura de la prevención. Se cierra el texto con un resumen de las principales conclusiones.

\section{Hacia una visión contextual-existencial del suicidio}

Es urgente un cambio en la conceptualización oficial del suicidio y en su modo de entender la prevención. El suicidio y las conductas suicidas, vistas desde la naturalización biomédica (García-Haro, García-Pascual, González González, Barrio-Martínez et al., 2020), conllevan un modelo muy claro de abordaje terapéutico y de prevención. Consiste en detectar y desactivar el factor diagnóstico. Para ello se acude al control de los síntomas a través de la intervención farmacológica.

Contra esta idea de una naturalización biomédica y diagnóstico-céntrica del suicidio, se propone aquí una visión abierta-contextual-existencial, plural y dinámico-fluctuante-interactiva del proceso y fenomenología suicidas.

1. El suicidio es una realidad abierta-contextual-existencial porque el ser humano, la vida humana o el comportamiento humano, no es una cosa hecha, una sustancia, una realidad acabada, sino un acontecer dramático (Ortega y Gasset, 1974), un tener-que- hacer-algo-para-vivir, para sobrevivir o incluso para morir. Este tener-que-hacer-algo, coincidiría con la estructura misma de la conducta (Yela, 1996) y con la definición de Psicología como ciencia que estudia lo que hace un sujeto con su 
comportamiento en una situación determinada para hacer su (proyecto de) vida. Como dice Yela, el sujeto humano no está simplemente entre el estímulo y la respuesta, sino que $v a$ a la situación con ciertas actitudes y disposiciones -pro-yectos, pro-pósitos, pre-juicios, pre-disposiciones, pre-tensiones, pre-visiones- en virtud de las cuales se sitúa en el mundo y confiere a la situación un significado (Yela, 1996). Este que-hacer, o proyecto vital, como diría Ortega y Gasset (1966), tiene lugar en una circunstancia determinada, la cual le ofrece al sujeto una serie de facilidades y resistencias. Cuando el balance posibilidades/dificultades es positivo, se navega hacia delante a pesar de todo. Cuando las adversidades se hacen insuperables se produce una crisis y naufragio existenciales, cae el sujeto de su embarcación de seguridad y queda braceando contra las olas del sufrimiento (vivido-subjetivo antes que clínico o diagnosticado-objetivo), desde cuyo pathos emerge la experiencia suicida.

2. Es una realidad plural porque el suicidio no es fenómeno homogéneo o unitario, susceptible de ser reducido a un núcleo o esencia, sino que se muestra como plural y diverso. Así cabe diferenciar entre el suicidio de niños y ancianos, hombres y mujeres, individuales y colectivos, altruistas y egoístas, asistidos y no asistidos, etc. El libro Suicidio(s) de Rendueles muestra claramente esta tesis (Rendueles, 2018).

3. Finalmente, el suicidio (así como muchos de los factores de riesgo) es una realidad dinámico-fluctuante (más que fija) susceptible de cambio de un día para otro o incluso en horas (Rudd, 2006). Esta variabilidad inherente al proceso suicida es la que impide hacer predicciones fiables (Chang et al., 2016; Franklin et al., 2017). Dicha manifestación dinámico-fluctuante del suicidio no sería así porque sí, si no que tendría su raíz en la realidad interactiva (más que natural-fija-ahí-dada) que le caracteriza, porque los valores, los proyectos, los significados y las relaciones humanas, entre ellas la relación con el clínico que trata de evaluar-ayudar, influyen en el modo de manifestación o aparecer del objeto que quiere estudiar (Pérez-Álvarez, 2018b; Pérez-Álvarez, 2020). Para algunos terapeutas centrados en soluciones, los primeros 5-10 minutos de la entrevista son muy importantes (Henden, 2005, 2008). Si hay un fenómeno clínico donde el olvido del principio de incertidumbre de Heisenberg puede tener consecuencias nefastas, este es sin duda el de la evaluación del riesgo suicida. El siguiente caso ilustra esta temática:

Julián tiene 53 años, está separado y tiene un hijo de 14 años. Hijo único de militar. Su infancia transcurrió sin problemas hasta que su padre sufre un accidente y es jubilado por problemas de movilidad. La presencia de un padre frustrado en casa trae una educación rígida y agresiva. Su adolescencia se convierte en un infierno de palizas cada vez que no cumplía con las "órdenes" del padre. La madre es una figura débil y se posiciona a favor del padre como una manera de evitar ser ella misma la víctima de la agresión. A los 14 años Julián empieza a fracasar en el colegio y a relacionarse con malas influencias como una manera de desafiar la autoridad paterna y como preparación para huir de casa. Inicia una juventud problemática, se inicia en el consumo de alcohol y marihuana hasta que a los 18 años marcha de casa. Abandona los estudios, vive en la noche y en las drogas. Trabaja de camarero y portero de discoteca. Desarrolla una actitud agresiva frente a desencuentros mínimos que siempre vive como provocaciones "chulescas" a su persona. Se mete continuamente en peleas que, afortunadamente, nunca tienen consecuencias legales. Posteriormente inicia un proceso de desintoxicación y, con gran esfuerzo, consigue dejar las drogas. La vida le va bien, se mantiene lejos de su familia, se hace empresario de hostelería (tiene un restaurante y un pub), gana dinero, se casa, tiene un hijo al que apenas ve pues vive para su trabajo. Por su ausencia y carácter explosivo, Marian, su mujer, decide separarse. Julián queda muy afectado pues ello implica que no puede ver a su hijo, el cual ya tiene 10 años. Reclama legalmente una custodia compartida y lo consigue. Cuando el hijo está a solas con él experimenta cierto pánico a no ser buen padre y, sobre todo, miedo a parecerse al suyo. Su hijo le recuerda bastante a sí mismo con esa edad. No sabe cómo manejar la situación, se siente superado, empieza a revivir las palizas que recibió, se llena de rabia, no entiende el porqué de su falta de cariño. Mientras tanto recibe varias denuncias de proveedores a los que lleva tiempo sin pagar. Inicia un proceso de juicios sin fin que agotan su resistencia. Empieza a tener problemas económicos, los amigos le fallan, siente que no puede confiar en nadie, su ex-mujer amenaza con la retirada de 
la custodia. En esta situación desarrolla síntomas depresivos reactivos con una intensa necesidad de llorar, sentimientos de vacío, problemas de sueño, no tolera la soledad, lucha para no recaer en las drogas. Su médico le da la baja y le receta una medicación antidepresiva y ansiolítica. Poco después de la baja recibe la noticia del fallecimiento de su padre, al que hacía años que no veía. Acude al funeral como un extraño. Lo pasa fatal, no soporta lo que llama la "hipocresía de la sociedad". Esa noche no duerme, siente una fuerte angustia, tristeza, ganas de llorar y de acabar con todo. Empieza a aislarse, a rumiar lo que ha sido su vida y su persona, no ve salida, no ve futuro, toma alcohol, coloca una cuerda en el salón de la casa con intención de quitarse la vida, entonces suena el teléfono. Es su hijo que le plantea que quiere pasar el fin de semana con él. Esta llamada a última hora modifica su plan suicida y funciona como una "razón para vivir". Una semana antes su Médico de Atención Primaria le había derivado a Salud Mental con la siguiente nota: "Depresión reactiva. Paciente de 50 años en situación de baja laboral, a tratamiento con Paroxetina y Tranxilium, en los últimos meses se encuentra peor, problemas laborales y familiares".

Tómese este caso para repensar el significado del tópico: el 90\% de las personas que se suicidan sufrían un trastorno mental. Parece obvio que, sin dejar de cumplir criterios de trastorno mental, Julián no intentó suicidarse porque sufría una depresión sino por la carga de sufrimiento que soportaba y la prospección de un aciago futuro.

La petición weberiana de separar en ciencia los hechos y valores, difícil de lograr en cualquier fenómeno psicológico, se hace imposible en el problema del suicidio. El acto de quitarse la vida es indisociable de los valores-proyectos de un sujeto, sea siquiera el valor que le da esa persona a su yo (indigno, no válido, no merecedor de aprecio), a su vida (no vale la pena vivirla) o a la muerte (trascendencia-fin del sufrimiento). Si hay un problema humano donde los valores y las preguntas existenciales se hacen patentes, ese es sin duda el del suicidio.

\section{Suicidio como drama existencial y la figura del iceberg}

Esta realidad contextual-existencial del suicidio, según la hemos presentado, se ajustaría a la figura de drama existencial (García-Haro, González González, Barrio-Martínez et al., 2020) con sus dos polos: la circunstancia de la vida con sus problemas, conflictos y dilemas, y la elección de posibilidades. Una de esas posibilidades es, en efecto, el acto suicida. Para una representación del drama existencial del suicidio piense el lector en la muerte de Adela en la obra La casa de Bernarda Alba (García Lorca, 1972), de Madama Butterfly (véase la lectura de Linares, 2020), de Ana Karenina (Tolstói, 1877/1977), de Madame Bovary (Flaubert, 1957/2019), etc. Invocar una etiqueta diagnóstica como explicación de estos suicidios es comprensible (se ajusta al modelo biomédico dominante en la cultura de salud mental) y tentador (supone un atajo cognitivo que simplifica y ahorra esfuerzo hermenéutico), pero desafortunado en la toprevención y en la ayuda, pues desatiende la complejidad de una biografía profundamente dañada por el sufrimiento existencial. Como dice Menéndez Osorio: "La clínica del sujeto es una clínica de las biografías, de lo singular, de las deferencias específicas, de los enunciados en primera persona, de la identidad narrativa" (Menéndez Osorio, 2020, p.15).

La imagen del iceberg sirve para ilustrar las dimensiones o capas del drama existencial del suicidio. La parte emergente (A) del iceberg serían los comportamientos autodestructivos (ideación suicida o autolesiva, planificación suicida o autolesiva, tentativas de suicidio, autolesión), tanto presentes como pasados. El aire que está en contacto con dicha parte emergente serían los síntomas y trastornos psicopatológicos (B), muy a menudo conductas depresivas y de consumo de alcohol. La parte sumergida (C) serían los procesos psicológicos subyacentes; el dolor psicológico y la desesperanza (Klonsky y May, 2015), la vivencia de humillación, derrota y atrapamiento (O’Connor, 2011), son importantes factores psicológicos que constituyen el fondo del suicidio. $\mathrm{El}$ agua de mar que circunda y mantiene mediante enfriamiento el bloque de hielo sumergido representa el drama de la vida personal (D) con sus conflictos y dilemas, con su biografía dañada, con su modo particular de construir el mundo y estilo de afrontamiento, con sus apoyos y soledad; todo un mundo de la vida, invisible a 
los ojos porque es demasiado ubicuo para ser visto. No en vano, el suicidio es un problema de "mi” vida. Aquí destacan los factores familiares y de apego, las experiencias adversas durante la infancia (ELA, Early-life adversity, por sus siglas en inglés), los contextos vitales problemáticos (García-Haro et al., 2018), las estrategias de afrontamiento, etc. Para una revisión de los principales ELA en la infancia y adolescencia asociados al suicidio véase Al-Halabí et al. (En prensa).

No es este el lugar para revisar las limitaciones de la investigación oficial (mainstream) del suicidio. Tan sólo se dirá que ha consistido, y consistirá, invariablemente, en calcular correlaciones p-significativas entre elementos de A con elementos de B. Difícilmente se avanzará en la comprensión del suicidio por esta vía, por más que se publiquen montañas de artículos en las revistas más prestigiosas. De forma coherente a la investigación, la evaluación clínica de la conducta suicida, centrada en la estimación del riesgo, ha pivotado, y pivotará, en torno a los dos primeros elementos, ignorando los dos últimos, especialmente el fondo del pathos o crisis existencial. Finalmente, el enfoque de intervención tradicional en el sistema sanitario (así como en la investigación de la efectividad de las terapias) se ha centrado, y se centrará, exclusivamente en la parte superficial, ya sea en los síntomas del trastorno o en los comportamientos suicidas en sí mismos como si fueran ellos mismos, en su topografía, el problema (control del riesgo y plan de seguridad), ignorando su funcionalidad contextual y la crisis vital-biográfica subyacente de la persona. Así, una persona puede ser dada de alta de una unidad de hospitalización porque "ya no presenta ideas de suicidio o niega intencionalidad suicida", y seguir sumergida, incluso más que antes de ingresar, en una dinámica suicida.

La imagen del iceberg ha sido utilizada en la literatura del suicidio desde otras perspectivas: 1) Epidemiológica: para señalar que por debajo de los suicidios consumados oficiales habría un elevado número de tentativas y de suicidios no registrados, y 2) Para reflejar la continuidad entre las autolesiones (parte sumergida del iceberg) y las conductas suicidas (Arensman et al., 2018; McMahon et al., 2014). Comparado con nuestra imagen, lo que estos dos modelos colocan en la parte sumergida sigue siendo para nosotros la parte más superficial del suicidio, su epidermis conductual.

\section{Recomendaciones para la prevención futura}

Desde esta óptica existencial-plural-interactiva del suicidio cabría ahora preguntarse qué es prevención del suicidio. Se darán sólo unas pinceladas en torno a tres puntos: el papel del control de síntomas, las razones para querer morir y los factores de protección.

1. Los Planes, Estrategias y/o Protocolos para la prevención y tratamiento del suicidio no deberían ser sólo campañas de detección y abatimiento de síntomas (intervención centrada en la parte superior del iceberg), sino que deberían ayudar a las personas en crisis a enfrentar los auténticos problemas y dilemas que la vida les plantea en primera persona, dotándoles de los recursos individuales y sociales necesarios. De lo contrario, una ayuda reducida a y reductora de síntomas mentales (apetito, sueño, ánimo, ideación suicida, etc.) no servirá para salir del drama existencial del suicidio. El control de síntomas, si nada cambia, contextual, familiar ni existencialmente, dará como resultado predecible un aumento del malestar y, correlativamente, un aumento del riesgo y de nuevos tratamientos, llegando hasta las medidas coercitivas como el ingreso involuntario, tan necesario como muchas veces evitable. Vale hacer notar aquí el uso, nada inocente ni casual, del término manejo de la conducta suicida o manejo de caso en lugar del de intervención o tratamiento. Apunta a una cuestión clave de los Planes, Estrategias y/o Protocolos. Se refiere a la primacía de una intervención centrada en tratar un cuadro clínico (modelo de intervención dominante) en lugar de ayudar a la persona a salir de su experiencia de atrapamiento (O'Connor, 2011) y su sufrimiento vivencial. Como han señalado Rogers y Soyka (2004), este abordaje-tipo centrado en la sintomatología produce un "efecto deshumanizante"; crea distancia entre la persona que busca ayuda y el clínico y por tanto aísla; construye una identidad suicida y por tanto un manto de vergüenza tras el que ocultarse; y finalmente, induce desesperanza e indefensión respecto de las posibilidades de solución. Véase que todos estos elementos (aislamiento, vergüienza, desesperanza, indefensión) son factores conductuales y cognitivos bien contrastados por 
la investigación empírica de aumento del riesgo suicida (véase el sistema de creencias suicidas dentro de la perspectiva de la teoría de la vulnerabilidad fluida, Bryan y Rudd, 2018).

Por lo tanto, la finalidad de los Planes, Estrategias y/o Protocolos de atención-prevención del suicidio no debiera consistir sólo en impedir la muerte (dispositivos de vigilancia, control farmacológico, adherencia e ingreso hospitalario, sin ser esto poco), sino en ayudar a las personas a engancharse a la vida, para lo cual se requiere no sólo saber lo que tiene (identificación y cuantificación de los comportamientos suicidas), sino entender lo que pasa; construir una narrativa biográfica de sentido (cómo hemos llegado hasta aquí) y de futuro (construir un horizonte de esperanza). Además, el enfoque centrado en síntomas y alarmas no siempre encaja en lo que las personas que acuden a consulta piensan que les ayudaría (Thomas y Leitner, 2005). Los objetivos basados en ayudar a la persona a salir del atrapamiento (O’Connor, 2011) y sufrimiento vivencial y de construcción de un futuro de esperanza encajarían perfectamente con las aportaciones de un trabajo social comunitario y psicoterapéutico en los centros de atención primaria y de salud mental. Por ahí debieran ir, a nuestro juicio, los esfuerzos de la prevención sanitaria del suicidio.

2. Habría que abordar no sólo los factores de riesgo o correlatos, biológicos o psicológicos, asociados a la conducta suicida (neurotransmisores, polimorfismos, colesterol, procesos inflamatorios, apatía, impulsividad, depresión, sesgos atencionales, cogniciones negativas, etc.), sino atender las "razones" o "racionalizaciones", verbalizadas o silenciadas, por las cuales una persona desea morir. Se precisa escuchar la narrativa suicida. A poco que se deje hablar al protagonista - y, con independencia de lo que se escuche (síntomas o problemas), cada vez se deja hablar menos-, emergerá una trama de contextos vitales problemáticos dentro de los cuales la persona se halla atrapada, enredada y desesperada, a menudo todo ello oculto bajo un manto de síntomas depresivos, los cuales muchas veces son ellos mismos más una consecuencia o correlato del atrapamiento (O'Connor, 2011) y sufrimiento vivido, que una causa del suicidio o disparadores del mismo. La circunstancia vivida es la que da sentido a la aparición y/o mantenimiento de los síntomas. No es el humo lo que hace que una persona salte desesperada de un edificio en llamas, sino la inminencia de un fuego abrasador que amenaza con quemarlo todo. Es por ello fundamental trabajar siempre con una hipótesis de por qué esa persona concreta a la que asistimos verbaliza deseos de morir o intenta quitarse la vida, más allá de caer en principios dormitivos (Bateson, 1972) según los cuales los diagnósticos (descriptivos) o los constructos teóricos explican las conductas, y las experiencias problemáticas. Si no se cuenta con una hipótesis (existencial-biográfica-familiar-contextual), la que se activa por defecto, sin pensar (sistema 1 según el modelo de Kahneman, 2012) o por habitus, según Bourdieu (Vázquez García, 2002), es la explicación diagnosticocéntrica. No es el objetivo de este trabajo profundizar en estas hipótesis. Para una aproximación véase Linehan (1993), Laso (2020) y Linares (2020).

3. Debería ser un imperativo asistencial de todo plan de prevención sanitaria potenciar los factores de protección, tanto individuales, como grupales y sociales, incluyendo los apoyos socio-emocionales (Sánchez-Teruel y Robles-Bello, 2014; Sánchez-Teruel et al., 2018), así como abordar los determinantes sociales que inciden en su manifestación (Navarrete Betancort et al., 2019). Este objetivo converge con el concepto de promoción de la salud mental positiva del Plan de Valencia (Sotoca Covaleda, 2018), si bien este Plan no da ninguna indicación de cómo lograr dicho objetivo-. Tendría una orientación universal, selectiva e indicada. Se trataría de un nuevo paradigma de prevención o al menos complementario al tradicional que no sólo se centra en abordar lo que va mal, sino que contempla la potenciación de los recursos y la promoción de la salud mental (Henden, 2005; Fiske, 2008; McAllister et al., 2009).

No dar prioridad a los aspectos sociales-contextuales en la prevención del suicidio puede ser un grave error de incalculable valor vital, pues la mejor -y acaso única- manera de salir con garantías de una crisis consiste, precisamente, en el doble movimiento de potenciar los recursos de las personas (véase las estrategias de afrontamiento, el sentido de pertenencia y conexión, la red de apoyo social, las razones para vivir, el sentido vital, los valores, los proyectos de futuro, etc.) y de mejorar, hasta donde sea posible, las condiciones reales de su existencia. Se trataría de salvar la circunstancia (Ortega y Gasset, 1960) para que la persona siga adelante con 
su vida sin ceder a la tentación de acabar con su yo. Lo anterior no es fácil, pero es fundamental para la prevención. Si aumentan las demandas de búsqueda de ayuda en salud mental es porque es más fácil medicalizar los problemas de la vida que cambiar las situaciones sociales que llevan a las personas a desarrollar problemas psicológicos. En nuestros días, medicalizar síntomas va camino de ser un imperativo asistencial, tal y como lo refleja la, cada vez más frecuente, petición desde Atención Primaria: Paciente atendido por psicólogo sin tratamiento, considero que debe ser visto por psiquiatría. $\mathrm{O}$, el no menos común: ansiedad no controlada farmacológicamente.

Entre los factores de protección, interesa resaltar, por su centralidad, la construcción de una buena relación terapéutica, especialmente en sus cuatro dimensiones: seguridad en el sistema terapéutico, conexión emocional con el terapeuta, enganche en el proceso terapéutico y compartir el propósito de la ayuda dentro de la familia (Escudero y Friedlander, 2019). Empoderar (dar poder psicológico) a la persona que bracea contra las olas del suicidio debería ser siempre un objetivo terapéutico prioritario. Este trabajo de potenciación de los factores de protección y de intervención social está sin embargo ausente en la mayoría de Planes, Estrategias y/o Protocolos, o en el mejor de los casos, ocupa un lugar políticamente correcto, escrito en el papel, pero sin dotación de recursos ni seguimiento de su aplicación.

La ayuda que emerge desde este planteamiento existencial-plural-interactiva del suicidio tendría unas características distintivas: 1) No se centraría en el tratamiento de la enfermedad mental -y acaso esto puede ser más parte del problema que de la solución-, sino en la circunstancia vital que hace que esa persona individual, concreta y única, se encuentre caída (en depresión), atrapada en una crisis (existencial) y con deseos de morir; 2) La protección a la salud no se limitaría a la marca y vigilancia de la intimidad sino que colocaría en el centro a la persona y la relación terapéutica; 3) La prevención no sería únicamente, o principalmente, control del riesgo y manejo de la seguridad sino potenciación de los deseos de vida, y 4) La intervención de enfermería no se agotaría en saber cómo le va a la gente con la medicación o cómo está tomando la medicación. A modo de apunte, interesa señalar que un nuevo modelo de ayuda, basado en el cuidado de las necesidades psicosociales y orientado a soluciones, y no sólo pegado al control del riesgo, es posible por parte del personal de enfermería. Véase McAllister et al. (2009).

En definitiva, un programa de prevención del suicidio debería ir a las cosas mismas, esto es, ir más allá de la epidermis conductual del suicidio (A y B del iceberg) y llegar hasta el drama existencial de las personas. La farmacología, con ser importante, no llega a la raíz del problema. Se trataría de ayudar a las personas a construir una vida que valga la pena ser vivida y no sólo a impedir la muerte, lo cual, aunque necesario, sería un vivir continuamente en el filo de la muerte, una suerte de cuidados paliativos del suicidio. Dicho de otra manera: una atención protocolaria que se felicita por impedir la muerte a corto plazo, puede tener un final trágico si no busca, además, potenciar la vida. Se trataría no sólo de impedir la conducta suicida como de ayudar a las personas a enfrentar el “duro oficio de vivir” (Alvarez, 2003, p. 3).

Dar cabida a esta nueva perspectiva de ayuda y prevención del suicidio requiere profundos cambios conceptuales y prácticos en salud mental; por ejemplo, una ampliación o revisión del concepto de salud, abierto a lo existencial-contextual (Pérez-Álvarez, 2018a) y no reducido al arreglo de "averías" biológico-cerebrales o mentales-cognitivo-psicodinámicas (Pérez-Álvarez, 2019). Desde la óptica de la investigación, se precisaría complementar las explicaciones del modelo biomédico (lógica natural-causal-explicativa-biológica o neurocientífica), con las explicaciones de vivencia, motivación y significado (lógica narrativa-comprensiva-biográfica), propias de una ciencia humana-holista-contextual (Pérez-Álvarez y García Montes, 2019). Se trataría, en definitiva, de apostar por un enfoque más contextual que individual-subjetivo o corporal-biológico y más centrado en comprender el sentido/función de las experiencias suicidas que en descubrir causas naturales explicativas de la conducta suicida.

Con todo, esta perspectiva contextual-existencial del suicidio es más complementaria que alternativa al enfoque tradicional. No renuncia al uso de fármacos ni a la hospitalización cuando se precisan. Tan sólo dice que estas ayudas, por sí solas, ni son curativas ni son efectivas a largo plazo, como a veces se proclaman. Es más, en su optimismo terapéutico reproducen actitudes perjudiciales y estigmatizantes (Rogers y Soyka, 2004), y no debieran ser las primeras opciones en la prevención, aunque muchas veces sean imprescindibles y hasta irrenunciables. 
Una atención seria y sincera a las crisis y conductas suicidas dentro del sistema sanitario debe ayudar a las personas a solucionar los problemas de su vida, atender a las razones para desear morir y potenciar los factores de protección. Esto desafía y desborda el estrecho margen del modelo diagnosticocéntrico, típicamente DSM-5 (APA, 2013). Se precisa un nuevo enfoque conceptual, más justo con la realidad existencial-plural-interactiva del proceso suicida. Un nuevo modo de pensar el suicidio que ayude al profesional a comprender el drama biográfico del suicidio, a aceptar que las personas puedan tener vivencias de muerte y suicidio a falta de otras perspectivas mejores, $\mathrm{y}$, sobre todo, a tolerar la angustia sin precipitarse en decisiones-actuaciones innecesarias o iatrogénicas: derivar sin contener, dar pautas de sentido común, cambiar medicación, ingresar, vigilar, controlar, etc. A menudo se cumple aquí el principio según el cual el mejor calmante para la angustia del clínico es prescribir o pedir que prescriban un fármaco sedativo al paciente, a sabiendas, muchas veces, salvo autoengaño, de que esa no es la solución.

Se necesita una lectura crítica respecto de la investigación tradicional del suicidio y avanzar hacia una aproximación plural, cualitativa y contextual-existencial, para la comprensión, prevención, desestigmatización y ayuda a las personas con riesgo suicida, supervivientes y familias. Para ello se precisa de la colaboración interdisciplinar y multiprofesional de los profesionales de la medicina, la enfermería, la psicología, trabajo social, la sociología y la antropología. Pero, sobre todo, se necesita un cambio en el habitus de pensar el suicidio.

\section{Conclusiones}

Es necesario un cambio en la conceptualización del suicidio y con ello en la prevención del mismo. El modelo predominante en la actualidad, el biomédico, desvela la pretensión de querer ahormar al ser humano y la realidad abierta-existencial-plural-fluida-interactiva del suicidio en un molde mecánico y patológico, respectivamente. Dichas características (abierta, plural y dinámico-fluctuante) son más propias de una visión del suicidio contextual-existencial.

El enfoque de intervención tradicional se centra exclusivamente en la clínica psicopatológica, o en los comportamientos suicidas en sí mismos, ignorando su funcionalidad contextual y la crisis vital-biográfica subyacente de la persona.

El modelo que se expone, el contextual-existencial, es complementario al enfoque tradicional. Este enfoque aboga por programas de prevención que vayan más allá de los síntomas y trastornos psicopatológicos y de los comportamientos autodestructivos. Se trataría de llegar hasta el drama existencial de las personas y desde ahí ayudar a que puedan construir una vida que valga la pena ser vivida, potenciando los factores de protección.

\section{Referencias}

Al-Halabí, S., García-Haro, J. M. y Gutiérrez, B. (En prensa). Conducta suicida. En E. Fonseca (Ed.), Manual de Tratamientos Psicológicos Eficaces: Infancia y Adolescencia. Pirámide.

Alvarez, A. (2003). El Dios Salvaje. El duro oficio de vivir. Emecé.

American Psychiatric Association (APA). (2013). Diagnostic and Statistical Manual of Mental Disorders-5 (5th ed.). Autor.

Arensman, E., Corcoran, P. y McMahon, E. (2018). The iceberg model of self-harm: new evidence and insights. The Lancet. Psychiatry, 5(2), 100-101. https://doi.org/10.1016/s2215-0366(17)30477-7

Bateson, G. (1972). Pasos hacia una ecología de la mente. Una aproximación revolucionaria a la autocomprensión del hombre. Ediciones Carlos Lohlé.

Bryan, C. J. y Rudd, M. D. (2018). Brief cognitive-behavioral therapy for suicide prevention. Guilford Press. Cha, C. B., Franz, P. J., Guzmán, E., Glenn, C. R., Kleiman, E. M., y Nock, M. K. (2018). Annual Research Review: Suicide among youth - epidemiology, (potential) etiology, and treatment. Journal of Child Psychology and Psychiatry, and Allied Disciplines, 59(4), 460-482. 
Chang, B. P., Franklin, J. C., Ribeiro, J. D., Fox, K. R., Bentley, K. H., Kleiman, E. M., y Nock, M. K. (2016). Biological risk factors for suicidal behaviors: a meta-analysis. Translational Psychiatry, 6(9), Artículo e887. https://doi.org/10.1038/tp.2016.165

Escudero, V. y Friedlander, M. L. (2019). Alianza terapéutica con familias. Cómo empoderar al cliente en los casos difíciles. Herder.

Fiske, H. (2008). Hope in action: solution-focused conversations about suicide. Routledge. https://doi.org/ 10.4324/9780203843819

Flaubert, G. (2019). Madame Bovary. Alianza. (Trabajo Original publicado en 1857)

Franklin, J. C., Ribeiro, J. D., Fox, K. R., Bentley, K. H., Kleiman, E. M., Huang, X., Musacchio, K.M., Jaroszewski, A.C., Chang, B.P., Nock, M. K. (2017). Risk factors for suicidal thoughts and behaviors: A meta-analysis of 50 years of research. Psychological Bulletin, 143(2), 187-232. https://doi.org/10.1037/ bul0000084

García Lorca, F. (1972). La casa de Bernarda Alba. Losada.

García-Haro, J., García-Pascual, H. y González González, M. (2018). Un enfoque contextual-fenomenológico sobre el suicidio. Revista de la Asociación Española de Neuropsiquiatría, 38(134), 381-400.

García-Haro, J., García-Pascual, H., González González, M., Barrio-Martínez, S. y García-Pascual, R. (2020). Para pensar el suicidio más allá de la enfermedad y los diagnósticos: un enfoque contextual-fenomenológico. Norte de Salud Mental, XVI(62), 42-51.

García-Haro, J., García-Pascual, H., González González, M., Sánchez Pérez, M. T. y Barrio-Martínez, S. (2020). Abordaje y prevención del comportamiento suicida en el sistema sanitario español: enfoque, límites y riesgos. Cuadernos de Psiquiatría Comunitaria, 17(1), 56-81.

González González, M., García-Haro, J. y García-Pascual, H. (2019). Evaluación contextual-fenomenológica de las conductas suicidas. Revista de la Asociación Española de Neuropsiquiatría, 39(135), 15-31. https://doi.org/10.4321/s0211-57352019000100002

Henden, J. (2005). Preventing suicide using a solution focused approach. The Journal of Primary Care Mental Health, 8(3), 81-88.

Henden, J. (2008). Preventing suicide. The solution focused approach. John Wiley \& Sons.

Khaneman, D. (2012). Pensar rápido, pensar despacio. Debate.

Klonsky, E. y May, A. (2015). The Three-Step Theory (3ST): A New Theory of Suicide Rooted in the "Ideation-to-Action" Framework. International Journal of Cognitive Therapy, 8(2), 114-129. https://doi.org/10.1521/ ijet.2015.8.2.114

Laso, E. (2020). El suicidio: La perspectiva de la clave emocional. Mosaico, 75, 82-103.

Linares, J. L. (2020). Más allá de la autodestrucción. El drama relacional del suicidio. Mosaico, 75, 36-39.

Linehan, M. M. (1993). Diagnosis and treatment of mental disorders. Cognitive-behavioral treatment of borderline personality disorder. Guilford Press.

McAllister, M., Billett, S., Moyle, W. y Zimmer-Gembeck, M. (2009). Use of a think-aloud procedure to explore the relationship between clinical reasoning and solution-focused training in self-harm for emergency nurses. Journal of Psychiatric and Mental Health Nursing, 16(2), 121-128. https://doi.org/10.1111/ j.1365-2850.2008.01339.x

McMahon, E. M., Keeley, H., Cannon, M., Arensman, E., Perry, I. J., Clarke, M., Chambers, D. y Corcoran, P. (2014). The iceberg of suicide and self-harm in Irish adolescents: a population-based study. Social Psychiatry and Psychiatric Epidemiology, 49(12), 1929-1935. https://doi.org/10.1007/s00127-014-0907-z

Menéndez Osorio, F. (2020). Suicidio: clínica o voluntad de morir. Cuadernos de Psiquiatría Comunitaria, 17(1), 10-26.

Navarrete Betancort, E. M., Herrera Rodríguez, J. y León Pérez, P. (2019). Los límites de la prevención del suicidio. Revista de la Asociación Española de Neuropsiquiatría, 39(135), 193-214.

O'Connor, R. C. (2011). Towards an integrated motivational-volitional model of suicidal behaviour. En R.C O'Connor, S. Platt y J. Gordon (Eds.), International Handbook of suicide prevention: research, policy and practice (pp. 181-198). John Wiley \& Sons. https://doi.org/10.1002/9781119998556.ch11

Ortega y Gasset, J. (1960). Meditaciones del Quijote. El Arquero/Revista de Occidente. 
Ortega y Gasset, J. (1966). ¿Qué es filosofía? El Arquero/Revista de Occidente.

Ortega y Gasset, J. (1974). Unas lecciones de metafísica. El Arquero/Revista de Occidente.

Pérez-Álvarez, M. (2018a). Giro transdiagnóstico y vuelta de la psicopatología: Propuesta de una integración existencial-contextual. FOCAD para División de Psicología Clínica enero-junio 2018. Consejo General de la Psicología de España.

Pérez-Álvarez, M. (2018b). Para pensar la psicología más allá de la mente y el cerebro: un enfoque transteórico. Papeles del Psicólogo, 39(3), 161-173. https://doi.org/10.23923/pap.psico 12018.2875

Pérez-Álvarez, M. (2019). La psicoterapia como ciencia humana, más que tecnológica. Papeles del Psicólogo, 40(1), 1-14. https://doi.org/10.23923/pap.psicol2019.2877

Pérez-Álvarez, M. (2020). El embrollo científico de la psicoterapia: Cómo salir. Papeles del Psicólogo, 41(3), 174-183. https://doi.org/10.23923/pap.psicol2020.2944

Pérez-Álvarez y García Montes, J. M. (2019). La filosofía de la ciencia aplicada a la psicología científica: pensando en psicosis. En E. F. Pedrero (Coord.), Tratamientos psicológicos para la psicosis (pp. 123-144). Pirámide.

Rendueles, G. (2018). Suicidio(s). Grupo 5.

Rogers, J. R. y Soyka, K. M. (2004). “One size fits all”: An existential-constructivist perspective on the crisis intervention approach with suicidal individuals. Journal of Contemporary Psychotherapy, 34, 7-22. https:// doi.org/10.1023/b:jocp.0000010910.74165.3a

Rudd, M. D. (2006). Fluid vulnerability theory: a cognitive approach to understanding the process of acute and chronic suicide risk. En T. E. Ellis (Ed.), Cognition and suicide: Theory, Research and Therapy (pp. 355368). American Psychological Association. https://doi.org/10.1037/11377-016

Sánchez-Teruel, D. y Robles-Bello, M. A. (2014). Factores protectores que promueven la resiliencia ante el suicidio en adolescentes y jóvenes. Papeles del Psicólogo, 35(2), 181-192.

Sánchez-Teruel, D., Muela-Martínez, J. A. y García-León, A. (2018). Variables de riesgo y protección relacionadas con la tentativa de suicidio. Revista de Psicopatología y Psicología Clínica, 23(3), 221-229. https:// doi.org/10.5944/rppc.vol.23.num.3.2018.19106

Sotoca Covaleda, R. (Dir). (2018). Vivir es la salida. Plan de Prevención del suicidio y manejo de la conducta suicida. Generalitat Valenciana http://www.prevenciodelsuicidi.san.gva.es/documents/7217942/7267322/ GU\%C3\%8DA+SUICIDIO+\%C3\%81MBITO+SANITARIO.pdf

Thomas, J. C. y Leitner, L. M. (2005). Styles of suicide intervention: Professionals' responses and clients' preferences. The Humanistic Psychologist, 33(2), 145-165. https://doi.org/10.1207/s15473333thp3302 5

Tolstoi, L. (1877). Anna Karenina. Juventud. (Trabajo original publicado en 1877).

Vázquez García, F. (2002). Pierre Bourdieu. La sociología como crítica de la razón. Montesinos.

Yela, M. (1996). La estructura de la conducta. Estímulo, situación y conciencia. Psicothema, 8(supl), 89-147.

Artículo recibido: 26/08/2020

Artículo aceptado: 23/02/2021 